\title{
Chemical composition of stellar populations in $\omega$ Centauri
}

\author{
A. F. Marino ${ }^{1,2}$, G. Piotto ${ }^{1}$, R. Gratton ${ }^{3}$, A. P. Milone ${ }^{1}$, M. Zoccali ${ }^{2}$, \\ L. R. Bedin ${ }^{4}$, S. Villanova ${ }^{5}$ and A. Bellini ${ }^{1,4}$ \\ ${ }^{1}$ Dept. of Astronomy, University of Padova, Vicolo dell'osservatorio 3, 35122, Padova, Italy \\ email: anna.marino@unipd.it \\ ${ }^{2}$ P. Univ. Católica de Chile, Dept. de Astronomía y Astrofísica, Casilla 306, Santiago 22, Chile \\ ${ }^{3}$ INAF-Osservatorio Astronomico di Padova, Vicolo dell'Osservatorio 5, 35122 Padova, Italy \\ ${ }^{4}$ Space Telescope Science Institute, 3700 San Martin Drive, Baltimore, MD 21218, USA \\ ${ }^{5}$ Dept. de Astronomia, Universidad de Concepcion, Casilla 160-C, Concepcion, Chile
}

\begin{abstract}
We derive abundances of $\mathrm{Fe}, \mathrm{Na}, \mathrm{O}, \alpha$ and $s$-elements from GIRAFFE@VLT spectra for more than 200 red giant stars in the Milky Way satellite $\omega$ Centauri. Our preliminary results are that: $(i)$ we confirm that $\omega$ Centauri exhibits large star-to-star metallicity variation $(\sim 1.4$ dex); (ii) the metallicity distribution reveals the presence of at least five stellar populations with different $[\mathrm{Fe} / \mathrm{H}] ;$ ( iii) a distinct Na-O anticorrelation is clearly observed for the metal-poor and metal-intermediate stellar populations while apparently the anticorrelation disappears for the most metal rich populations. Interestingly the $\mathrm{Na}$ level grows with iron.
\end{abstract}

Keywords. galaxy: stellar content, Globular clusters: individual (Omega Centauri)

\section{Introduction}

Omega Centauri ( $\omega$ Cen) is among the most studied and enigmatic Milky Way satellites. It has always been considered a Globular Cluster (GC), but a number of peculiarities, like the mass, the kinematics, and the complexity of its numerous populations identified by both spectroscopic and photometric investigations (Cannon \& Stobie 1973, Lee et al. 1999, Pancino et al. 2000, Bedin et al. 2004, Piotto et al. 2005, Villanova et al. 2007), suggest that it may be the remnant of a larger stellar system.

Recently, it has been shown that some peculiar features observed in $\omega$ Cen are shared with other GCs: A bimodality in s-elements is present in NGC 1851 (Yong et al. 2008) and M22 (Marino et al. 2009), and intrinsic variations in $[\mathrm{Fe} / \mathrm{H}]$ were detected in M22 (Marino et al. 2009), and M54 (Sarajedini \& Layden 1995; Bellazzini et al. 2008; Carretta et al. 2010). However $\omega$ Cen still remains a unique GC in the Milky Way as concerns the complexity of its stellar populations.

Here we present preliminary results of our project aimed to characterize the evolutionary connections of the sub-populations in $\omega$ Cen, by studying their chemical content.

\section{Observations and data reduction}

We analyzed FLAMES/GIRAFFE HR09 and HR13 spectra for a sample of more than 200 red giant stars. Iron abundances are obtained from an equivalent width analysis by using the Local Thermodynamical Equilibrium program MOOG (Sneden 1973), while the other elements are measured by comparing observed spectra with synthetic ones. More details on the abundance measurements can be found in Marino et al. (2008, 2009). 


\section{Results}

We obtained that the $[\mathrm{Fe} / \mathrm{H}]$ ranges from $\sim-2.1$ to $\sim-0.7$ dex, with at least five distinct stellar groups in the iron distribution as shown in Fig. 1 (left panel). In the top right panels of Fig. 1 we represent $\mathrm{Na}$ and $\mathrm{O}$ abundances for the five sub-populations, selected on the basis of different iron content and position on the color magnitude diagram (CMD). In the lower panels the position on the $B-(B-R)$ CMD from Bellini et al. (2009) for the different selected groups is shown, with the corresponding $\mathrm{NaO}$ anticorrelation in each upper panel. We superimposed to each Na-O plane, a fiducial traced by hand on the $\mathrm{NaO}$ anticorrelation for stars with $-1.80<[\mathrm{Fe} / \mathrm{H}]<-1.34$. We note that the $\mathrm{NaO}$ anticorrelation is well defined for stars belonging to the metal intermediate populations $(-1.80<[\mathrm{Fe} / \mathrm{H}]<-1.34)$, and some hints of a probably less extended one are present for the more metal poor stars with $[\mathrm{Fe} / \mathrm{H}]<-1.80$. Apparently, the anticorrelation disappears for stars with $[\mathrm{Fe} / \mathrm{H}]>-1.00$. Note that the Na level grows with Fe.

From the analysis of the s-elements La and $\mathrm{Ba}$ we derive that the s element abundance grows with increasing iron, and interestingly enough, in each group defined as in Fig. 1, apparently the s element abundance increases also with Na.
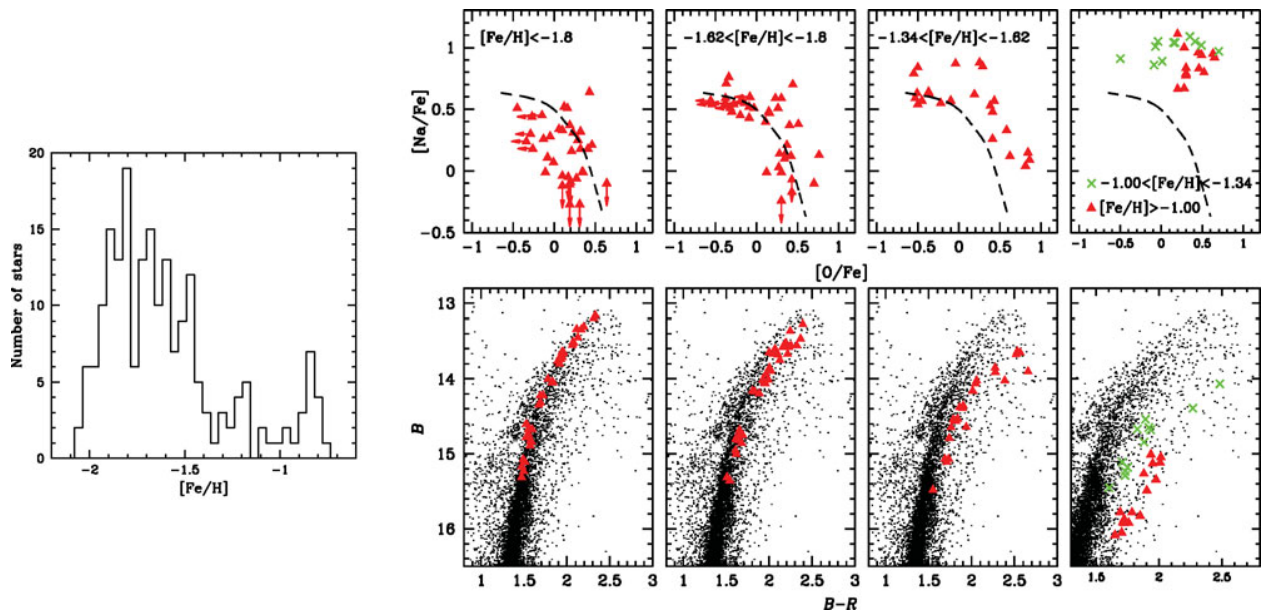

Figure 1. Left panel: Distribution of the iron content for our analyzed sample. Right panels: $\mathrm{Na}-\mathrm{O}$ anticorrelation for the different groups of stars selected on the basis of the iron content and the position on the CMD represented in lower panels.

\section{References}

Bedin, L. R., et al., 2004, ApJ, 605, L125

Bellazzini, M., et al., 2008, AJ, 136, 1147

Bellini, A., et al., 2009, A\&A A, 493, 959

Cannon, R. D. \& Stobie R. S., 1973, MNRAS, 162, 207

Carretta, E., et al., 2010, ApJL in press

Marino, A. F., et al., 2008, A\&SA, 490, 625

Marino, A. F., et al., 2009, A\&A, 505, 1099

Lee, Y.-W., et al. 1999, Nature, 402, 55

Pancino, E., et al., 2000, ApJ, 534, L83

Piotto, G., et al., 2005, ApJ, 621, 777

Sarajedini, A. \& Layden, A. C., 1995, AJ, 109, 1086

Sneden, C. A., 1973, Ph.D. Thesis

Villanova, S. et al., 2007, ApJ, 663, 296 